\title{
Diagnostic difficulties in Glucokinase Hyperinsulinism
}

T. Meissner $^{1^{*}}$, J. Marquard $^{1}$, N. Cobo-Vuilleumier ${ }^{2}$, M. Maringa ${ }^{3}, P$. Rodríguez-Bada ${ }^{2}$, M. A. García-Gimeno ${ }^{4}$, M. J. Castro ${ }^{2}$, J. C. Aledo ${ }^{2,5}$, Elena Baixeras $^{2}$, J. Weber ${ }^{6}$, K. Olek ${ }^{7}$, P. Sanz ${ }^{4}$ E. Mayatepek ${ }^{1}$ and A. L. CuestaMuñoz $^{2^{*}}$

${ }^{1}$ Department of General Pediatrics, Universtity Children's Hospital Düsseldorf, Gemany.

${ }^{2}$ IMABIS Foundation and Centre for the Study of Pancreatic beta cell Diseases. Carlos Haya University Hospital, Málaga, Spain.

${ }^{3}$ Human Genetics Practice, Bonn, Germany.

${ }^{4}$ Institute of Biomedicine of Valencia (CSIC), Valencia, Spain.

${ }^{5}$ Molecular Biology and Biochemistry Department. University of Málaga, Málaga, Spain.

${ }^{6}$ Integragen, GMBH, Bonn, Germany

${ }^{7}$ Laboratory for Parentage Certificate, Gelsenkirchen, Germany

${ }^{*}$ Address correspondence and reprint requests to Thomas Meissner, M.D. at Department of General Pediatrics. University Children's Hospital. Moorenstr. 5, D40225 Düsseldorf, Germany. Phone: 49 211-8117687. Fax:49 211-8119512. E-mail: thomas.meissner@med.uni-duesseldorf.de

Antonio L. Cuesta-Muñoz MD, PhD. at IMABIS Foundation and Center for the Study of Pancreatic Beta-cell Diseases. Carlos Haya University Hospital, Avda. Carlos Haya n 82, 29010 Málaga, Spain. Phone: 34951291446. Fax: 34952614012 Email: alcm@fundacionimabis.org 


\section{Abstract}

Glucokinase Hyperinsulinism (GCK-HI) is a rare variant of congenital hyperinsulinism caused by activating mutations in the glucokinase gene resulting in overactivity of glucokinase within the pancreatic $\beta$-cell. Until now seven families with this condition have been described. Here we report on a new patient presenting already with increased birthweight and neonatal hypoglycemia. The patient was initially diagnosed to suffer from transient hyperinsulinism and was reevaluated at the age of 3 years with developmental delay. Morning glucose after an overnight fast was 2.5-3.6 mmol/l. Fasting tests revealed supressed insulin secretion at the end of fasting $(1.4-14.5 \mathrm{pmol} / \mathrm{l})$. Mutational analysis showed a novel heterozygous missense mutation in exon 10 c.1354G >C (p.Val452Leu). Functional characteristics of GK-V452L clearly illustrated overactivity of this mutation after expression in Escherichia coli as a glutathionyl S-transferase (GST) fusion protein. On the basis of diagnostic difficulties in this patient we reviewed the literature for diagnostic criteria for GCK-HI. There was no single consistent diagnostic criterion found. Insulin concentration at hypoglycemia, fasting test as well as reactive hypoglycemia after an oral glucose tolerance test were not conclusive for all patients. Particularly adults with milder symptoms of hypoglycemia as episodes of extreme hunger or sweatiness were only identified genetically within the family screening.

Therefore GCK-HI as an autosomal dominat inherited condition might be underestimated. Mutational analysis of the GCK-gene should be performed in all individuals with unclear episodes of hypoglycemia even without documented hyperinsulinism during hypoglycemia after fasting. Delay of diagnosis might results in mental handicaps of the affected individuals. 


\section{Keywords}

Congenital hyperinsulinism, Monogenic Hyperinsulinism, Glucokinase, Glucose stimulated insulin secretion (GSIS)

\section{Abbreviations}

Congenital hyperinsulinism HI

Glukokinase GK

Glucokinase gene GCK

Glucokinase hyperinsulinism GCK-HI

Glucose-stimulated insulin secretion GSIS

Oral glucose tolerance test OGTT 


\section{Introduction}

Congenital Hyperinsulinism (HI) is a heterogenic group of genetic disorders of the pancreatic $\beta$-cells characterized by recurrent episodes of hypoglycemia due to an inappropriate secretion of insulin (1-2). Abnormalities in several different genes have been found to be responsible for about $55 \%$ of the cases of $\mathrm{HI}$ (3). However, the molecular etiology of the remaining $45 \%$ remains unknown.

Mutations in the $\beta$ cell sulfonylurea receptor (SUR1, ABCC8) are the most common cause of $\mathrm{HI}$, responsible for $45 \%$ of cases (4). Other genes involved include the inward cell rectifying potassium channel (Kir6.2, KCNJ11) (5), Glutamate dehydrogenase (GLUD1) (6), short chain 3-hydroxy-acyl-CoA dehydrogenase (SCHAD, HADHSC) (7) and Glucokinase (GCK) (8). The latter appears to be a very rare cause of $\mathrm{HI}$, with only seven cases described to date in the world literature (8-12). $\mathrm{HI}$ is an extremely heterogeneous disorder in all aspects, including clinical presentation, morphology and genetics. Since the hypoglycemia associated with the disease can cause permanent neurological damage (13), both prompt diagnosis and early treatment are essential.

The glycolytic enzyme Glucokinase (GK) functions as the "glucose sensor" in the pancreatic $\beta$-cells and as such regulates glucose stimulated insulin secretion (GSIS) (14). The structural integrity of GK is crucial for the maintenance of normal glucose homeostasis. Activating mutations in glucokinase gene $(G C K)$ result in $\mathrm{HI}(G C K-H I)$, both mild and severe forms (812,14).

Here we describe a new patient with GCK-HI due to a de novo mutation in GCK. We also demonstrate diagnostic difficulties of this disorder for this 
patient and the so far published individuals. GCK-HI might be misdiagnosed as "unclear" or "idiopathic" hypoglycemia, or as transient hyperinsulinemic hypoglycemia, due to these diagnostic problems.

\section{Case Report}

The child was the first offspring of healthy non-consanguineous German parents. Family history was negative for hyperinsulinism or diabetes. There was marked macrosomia of the newborn with a birth weight of $5860 \mathrm{~g}$ and a length of $60 \mathrm{~cm}$. Recurrent low blood glucose levels were measured in the first day of life. At the third day of life the male newborn was admitted to the ICU of the local children hospital due to hypoglycemia and was treated with i.v. glucose. Hyperinsulinism was suspected. At 14 days of life the i.v. glucose was stopped and changed completely to oral carbohydrates in addition to frequent feeding. The patient was finally discharged with diagnosis of "transient hyperinsulinism" without special diet or medication and with glucose concentrations of $2.6-3.3 \mathrm{mmol} / \mathrm{l}$. At the age of 3 years he was sent to the neurology outpatient clinic of our hospital for evaluation of speech delay and mental retardation. The patient's mental state was evaluated with SnijdersOomen Non-verbal Intelligence test (SON-R 2 1/2 -7) and revealed an IQ of 86 with marked problems in his concentration ability. Glucose monitoring on the ward revealed persistence of low glucose concentrations $(1.7-4.3 \mathrm{mmol} / \mathrm{l})$ with low glucose levels particularly after the night fast (Figure 1). The patient was evaluated by fasting test and oral glucose tolerance test (OGTT) (Figure 2, Figure 3). Hyperinsulinism was suspected because of the neonatal history and mutational analysis was performed for the so far described genes for $\mathrm{HI}$ (ABCC8, KCNJ11, GCK, GLUD1). The patient was discharged with dietary 
recommendations. After diagnosing GCK-HI a treatment with diazoxide was started $(6-8 \mathrm{mg} / \mathrm{kg} / \mathrm{d})$. Diazoxide was not futher increased because next to the hypertrichosis as side effect parents reported a loss of appetite. A repeat of the SON-R 2,5-7 intelligence test at 4 years and 3 month revealed now an IQ of 105. Today at 4 years and 9 month of age the patient is able to bicycle and to swim. Despite diazoxide $(7 \mathrm{mg} / \mathrm{kg} /$ day $)$ his fasting glucose often drops again to 2.0-2.5 $\mathrm{mmol} / \mathrm{l}$ and diazoxide was increased again. However, the opinion of parents and medical staff is that the patient markedly improved after the start of diazoxide treatment.

\section{Laboratory methods}

Sequencing of the glucokinase gene

After extracting genomic DNA from peripheral lymphocytes of the proband all coding exons and exon/intron boundaries of the ABCC8-, KCNJ11-, GCK- and GLUD1-gene were amplified by PCR, purified and sequenced at the IntegraGen laboratory Bonn using the Big_Dye Terminator v3.1 cycle sequencing kit and the ABI PRISM DNA Analyzer 3730. Subsequently DNA samples of the patients parents were checked for the presence of the identified GCK mutation. In order to confirm the identified mutation all coding exons and exon/intron boundaries of GCK gene were amplified by PCR, purified and sequenced at Integragen laboratories using the Big Dye Terminator kit (PerkinElmer Wellesley, MA, USA) and the ABI PRISM DNA Analyzer 3700 (PerkinElmer Wellesley, MA, USA). The variant detected in exon 10 of GCK was identified by direct sequencing and verified by digestion with the restriction endonuclease Bbvl. 
Glucose and insulin measurements:

A standard $(1.75 \mathrm{~g} / \mathrm{kg})$ OGTT was performed after $12 \mathrm{~h}$ of overnight fasting. Blood samples for the determination of plasma glucose and serum insulin were drawn at $0,30,60,120$ and $160 \mathrm{~min}$. Plasma glucose during the OGTT was measured with a hexokinase method using a Roche/Hitachi® device. The glucose monitoring on the ward was also performed with a glucose oxidase method using a ecoSolo $I \cong$ by care diagnostica ${ }^{\circledR}$ Germany. Serum insulin at the OGTT and the fasting test was measured with the electrochemiluminescence immunoassay "ELICA" on a cobas $®$ e immunoassay analyzer by Roche Diagnostics $®$ Mannheim, Germany.

Site-directed mutagenesis and functional analysis of the GK protein

The methods have been described before in detail (15). The V452L mutation of the GCK gene was introduced into the wild type human pancreatic GCK using the Quick Change site-directed mutagenesis kit from Stratagene (La Jolla, CA). Plasmid pUC-GkB was used as a template in PCR reactions. The mutant was sequenced to confirm that only the desired mutation was introduced. The mutated plasmid (pUC-GkBV452L) was digested with EcoRI and Sall. Subsequently, the insert was sub-cloned into plasmid pGEX-6P-1 (Amersham Pharmacia) to allow its expression in Escherichia coli as a glutathionyl S-transferase (GST) fusion protein and its purification by affinity chromatography using GSH-sepharose beads. The purified recombinant GSTGK protein was routinely screened for purity by SDS-PAGE. Studies of the kinetic properties of the wild type protein (GK-WT) and GK-V452L were 
performed spectrophotometrically as described previously (15). The kinetic assays were performed in a tuneable VERSAmax microplate reader. We used non-linear kinetics according to the Hill equation to determine the affinity of the enzyme for glucose. In order to measure the glucose phosphorylation capacity of the enzyme we used the relative activity index, which was calculated according to the formula previously reported (9).

\section{Results}

The direct sequencing of GCK of the proband revealed a novel heterozygous missense mutation in exon 10 c.1354G>C, p.Val452Leu (V452L). This variant was not found in any other family members or in 100 healthy control chromosomes. Since the mutation was not found in the parents and the paternity test revealed parenthood of the parents tested the mutation V452L was considered as "de novo".

The results of functional studies of GK-WT and GK-V452L are presented in Table 1. The $\mathrm{S}_{0.5}$ of GK-V452L obtained was two fold lower than that for GKWT. The Hill coefficient decreased moderately, indicating lower cooperativity of the enzyme. The turnover rate of GK-V452L tended to be lower than that of GKWT, however the efficacy $\left(\mathrm{Kcat} \mathrm{S}_{0.5}\right)$ of the GK-V452L was almost two fold higher. The affinity of GK-V452L for its second substrate, MgATP ${ }^{2-}$, was the same as that for GK-WT. The relative activity index, as an expression of $\beta$-cell glucose usage of the enzyme, was markedly increased for GK-V452L (Table 1). These kinetic characteristics suggest that GK-V452L was the cause of the hyperinsulinism in the patient. 
In order to study the impact of GK-V452L on the threshold for GSIS, and therefore on glucose homeostasis we used a previously described mathematical model that takes into account the impact of blood glucose levels on the expression of GK for both alleles (15). The threshold for GSIS predicted by this model for GK-V452L was $1.9 \mathrm{mmol} / \mathrm{l}$ in contrast to the physiological threshold of $5 \mathrm{mmol} / \mathrm{l}$ (Table 1, Figure 4). This model illustrates the physiological consequences of the GK-V452L protein on GSIS and therefore on glucose homeostasis. When compared to a similar analysis of other disease-causing GCK mutations, this novel mutation is ranked similar to A456V and G68V (Fig. $3)$.

The oral glucose tolerance test (OGTT) presented glucose values of $1.8 \mathrm{mmol} / \mathrm{l}$ at 160 min with, interestingly, also low insulin values (Figure 3). Glucose monitoring in our patient showed recurrent low glucose concentrations (1.7-4.3 $\mathrm{mmol} / \mathrm{l})$ particularly after the night fast (Figure 1). The fasting test revealed different suppressed insulin concentrations (1.4-14.5 pmol/l) at 14,16 and 18 hours of fasting without severe hypoglycemia (lowest glucose concentration at $18 \mathrm{~h}$ was $2.2 \mathrm{mmol} / \mathrm{l}$ ), and again with low insulin values of $1.4 \mathrm{pmol} / \mathrm{l}$ (Figure 2).

\section{Discussion}

The critical role played by GK in the maintenance of glucose homeostasis is demonstrated by the fact that mutations in GCK will lead to a monogenic metabolic disease of the pancreatic $\beta$-cell. Indeed, heterozygous and homozygous inactivating mutations result in a type of monogenic diabetes known as maturity onset diabetes of the young 2 (MODY2) (16), and permanent neonatal diabetes (17) respectively, while activating mutations result in $\mathrm{HI}$ 
(GCK-HI) (8). There are only seven activating GCK mutations described and found in six families and one individual $(8-12,18)$ (Table 2). The clinical phenotype of this type of monogenic hyperinsulinism turned out to be highly heterogeneous, resulting in both mild and severe forms $(8-12,18)$.

Our proband is the second patient diagnosed with GCK-HI who presents a "de novo" GCK activating mutation and also has mental retardation. However, in two other unrelated patients with GCK-HI previously described, the GCK activating mutation was also considered "de novo" in one of their parents. Therefore, if we consider four "de novo" GCK activating mutations out of eight described so far, the probability of "de novo" activating mutation in GCK is importantly high (50\%).

Although the prevalence of GCK-HI today is very low, the high frequency of "de novo" activating mutations in this gene and the heterogeneity in both age of onset and clinical presentation, lead to potential diagnostic difficulties of GCK-HI. The diagnostic criteria for $\mathrm{HI}$ are mainly the increased carbohydrate intake needed to prevent hypoglycemia and elevated insulin concentrations during hypoglycemia that demonstrate a lack of the physiological suppression of insulin secretion at low glucose concentrations. In transitory hyeprinsulinism, the blood glucose levels are usually stabilized after treatment with carbohydrate intake and intravenous glucose infusion, and patients are discharged with dietary recommendations. Our patient was treated with intravenous glucose only for the first two weeks of life, and then was discharged, hence the diagnosis of "transient hyperinsulinism". The clinical manifestation of GCK-HI in the neonatal periode is heterogeneous. Indeed, of five patients with GCK-HI 
diagnosed in the neonatal period, including our proband, only in two cases the hyperinsulinism was permanent $(9,11)$. In the three other cases of $\mathrm{GCK}-\mathrm{HI}$, one patient was asymptomatic (10) and two treated with intravenous glucose infusion and discharge on standard formula milk (10) and with no medication, like in our proband. These three last cases, therefore, were susceptible of being diagnosed as "transient hyperinsulinism". In all these patients with GCK-HI diagnosed as transitory neonatal hyperinsulinism, the blood glucose levels will be always low with the risk of neurological damage later in life. In infants feeding at four weeks of age is usually done every four hours, whereas the fasting episode will be prolonged during the first year of life up to eleven hours. This might cause a worsening of fasting hypoglycemia. In fact, the patient who was asymptomatic presented at the age of 15 years with seizures. The second patient presented a more severe hyperinsulinemic hypoglycemia at infancy, and our patient presented the hyperinsulinemic hypoglycemia in the childhood with speech delay and mental retardation.

The consideration of this heterogeneity in the clinical manifestation of $\mathrm{GCK}-\mathrm{HI}$ in the neonatal period is highly important since any transitory neonatal hyperinsulinism can be in fact a case of transitory $\mathrm{GCK}-\mathrm{HI}$ that will appears again later in life and, with very high probability, with neurological damage due to the long hypoglycemia period (13). None of these patients were genetically tested at the moment of the first diagnosis of hyperinsulinemic hypoglycemia, consequently, both prompt genetic analysis and diagnosis as well as early treatment are critical to avoid the neurological damage. Furthermore in patients with seizures of unknown origin or in individuals with episodes of extreme hunger and symptoms of hypoglycemia, learning problems or development 
delay, fasting hypoglycemia should be ruled out. This is not only important in childhood since many of the GCK-HI individuals reported today were diagnosed as adults by family screening (Table 2 ). In some relatives $(8,9,10,12)$ of affected individuals, a screening for GCK-mutation was performed and almost all of the GCK-mutation positive identified family members had a positive medical history for hypoglycaemic symptoms. Only two of them had never hypoglycaemic symptoms in history (Table 2; B1 and D1) but they had a pathological fasting test (Table 3, Figure 5) and a pathological OGTT (Figure 6, see individual D1)

In patients with $\mathrm{HI}$ diagnosed after the neonatal period, the insulin concentrations during fasting test or at spontaneous hypoglycemia are usually elevated. However, the evaluation of insulin concentration in GCK-HI patients revealed that the insulin values are not always elevated at hypoglycemia (Table 3 , Figure 5). In fact, in our patient the insulin concentration dropped to a value of $1.4 \mathrm{pmol} / \mathrm{l}$ with a glycemia of $2.2 \mathrm{mmol} / \mathrm{l}$. Likewise, OGTT in our patient showed low insulin secretion at 120 and 160 minutes (Figure 3). The low insulin secretion found in many GCK-HI patients at the time of hypoglycemia is probably due to the effect of the GCK-mutation on the glucose liver metabolism. Since hepatic and pancreatic glucokinase are encoded by a single gene with two distinct cell-specific promoters hepatic mutated GK will be activated in GCK-HI. $99 \%$ of the GK enzyme has been proposed to reside in the hepatoparenchymal cells. There it serves a critical role in postprandial glucose clearing from circulation [19]. Overactivity of liver GK will result in enhanced glycolysis despite low glucose concentrations. At a point of time, when insulin levels are already low in a fasting situation or postprandial state this might further trigger hypoglycemia. 
Pharmacological treatment of GCK-HI patients is also difficult to establish. Indeed, although early studies suggested that GCK-HI is a mild form of $\mathrm{HI}$ with good respond to diazoxide in low or medium dosage $(8,9)$, in a more severe case of GCK-HI, treatment with high dosage of diazoxide $(20 \mathrm{mg} / \mathrm{kg} / \mathrm{day})$ in combination with chlorothiazide and nifidepin did not result in euglycemia (10). In a patient recently reported (12), the response to low dosage of diazoxide was limited and additional octreotide was used. Though our patient showed an initial response to diazoxide in a medium dosage $(8.0 \mathrm{mg} / \mathrm{kg} / \mathrm{day})$, he had again recurrent episodes of hypoglycemia after the night fast with nearly the same dosage $(6.8 \mathrm{mg} / \mathrm{kg} / \mathrm{d})$ later. Data about octreotide treatment in GCK$\mathrm{HI}$ are very limited. In one patient octreotide was stopped because of the frequent injections needed without using continuous subcutaneous pumping system (10). The insufficient response of patients to drugs inhibiting insulin secretion might be due to the $\beta$-cell-independent effect of glucokinase overactivity in the liver and corresponding increased hepatic glucose clearence.

In summary, most individuals with GCK-HI did not need any treatment despite dietary intervention when they present a mild phenotype. There might be a good response to diazoxide at low to medium dosage in the more severe affected individuals, but there are also some patients that do not completely respond to diazoxide at least in medium concentrations. Higher doses of diazoxide are sometimes not accepted by the parents of patients because of severe hypertrichosis, but there might be also other factors as the loss of appetite reported in our patient. Furthermore the same dosage may be less effective over the time (9). 
The outcome of the patients that are known today is also highly variable. Some individuals never had any symptoms of hypoglycemia and without pathological neurology despite recurrent fasting hypoglycemia $(8,9)$. From the 24 patients reported so far one patient was severely handicapped with mental retardation and epilepsy (11) and three had minor problems as learning difficulties or learning handicap (12). Our patient clearly improved clinically from diazoxide treatment. His concentrativeness was much better and whereas he was initially tested as mentally retarded at follow up he did caught up and was completely normal tested.

In conclusion, GCK-HI is a rarely identified condition. This might be due to the often mild symptoms, the difficulties in diagnosing this disorder and the limited awareness for GCK-HI. The high frequency of spontaneous mutation in patients with GCK-HI along with the high risk of developing mental retardation, hypoglycemic seizures, or recurrent symptoms of hypoglycemia means that any newborn with hypoglycemia of unknown origin not resolving in the first weeks of life, or with transient hyperinsulinism, should be tested for GCK mutations. Furthermore mutational analysis of the GCK gene has to be included also in older children, as well as in adults if fasting hypoglycemia of unknown origin has been found. Identification of up to now not diagnosed patients will help to treat these patients properly by diet or medication. 


\section{Acknowledgement}

MEC (P.I3); Dirección General de Investigación Científica y Técnica (SAF2005-08014; SAF2006-12863). Junta de Andalucía (SAS/PI-024/2007). Spanish Novo Nordisk Pharma grant and Instituto de Salud Carlos III (RCMN C03/08 and 02/PI021473) to ALCM.

\section{References}

1. Meissner T, Beinbrech B, Mayatepek E. Congenital hyperinsulinism: molecular basis of a heterogeneous disease. Hum Mutat. 1999;13:351361

2. Lindley KJ, Dunne MJ. Contemporary strategies in the diagnosis and management of neonatal hyperinsulinaemic hypoglycaemia. Early Hum Dev. 2005;81:61-72.

3. Aynsley-Green A, Hussain K, Hall J, Saudubray JM, Nihoul-Fekete C, De Lonlay-Debeney $\mathrm{P}$, Brunelle $\mathrm{F}$, Otonkoski T, Thornton P, Lindley KJ Practical management of hyperinsulinism of infancy. Arch Dis Child Fetal Neonatal. 2000;82:98-107

4. Thomas PM, Cote GJ, Wohllk N, Haddad B, Mathew PM, Rabl W, Aguilar-Bryan L, Gagel RF, Bryan J. Mutations in the sulfonylurea receptor gene in familial hyperinsulinemic hypoglycemia of infancy. Science. 1995;268:426-429

5. Nestorowicz A, Inagaki N, Gonoi T, Schoor KP, Wilson BA, Glaser B, Landau H, Stanley CA, Thornton PS, Seino S, Permutt MA. A nonsense mutation in the inward rectifier potassium channel gene, Kir6.2, is associated with familial hyperinsulinism. Diabetes. 1997;46:1743-1748

6. Stanley CA, Lieu YK, Hsu BY, Ming JE, Glaser B, Poncz M. Hyperinsulinism and hyperamonemia in infants with regulatory mutations of the glutamate dehydrogenase gene. N Engl J Med. 1998;338:13521357

7. Clayton PT, Eaton S, Aynsley-Green A, Edginton M, Hussain $\mathrm{K}$, Krywawych S, Datta V,. Malingré HEM, Berger R, van den Berg IET. Hyperinsulinism in short-chain l-3-hydroxyacyl-CoA dehydrogenase 
deficiency reveals the importance of ß-oxidation in insulin secretion. $J$. Clin. Invest. 2001;108:457-465.

8. Glaser B, Kesavan P, Heyman M, Davis E, Cuesta A, Buchs A, Stanley CA, Thornton PS, Permutt MA, Matschinsky FM, Herold HC. Familial hyperinsulinism caused by an activating glucokinase mutation. $N$ Engl J Med. 1998;338:226 -230

9. Christesen HB, Jacobsen BB, Odili S, Buettger C, Cuesta-Munoz A, Hansen T,Brusgaard K, Massa O, Magnuson MA, Shiota C, Matschinsky FM, Barbetti $F$. The second activating glucokinase mutation (A456V): implications for glucose homeostasis and diabetes therapy. Diabetes. 2002;51:1240-1246

10. Gloyn AL, Noordam K, Willemsen MA, Ellard S, Lam WW, Campbell IW, Midgley $P$, Shiota $C$, Buettger C, Magnuson MA, Matschinsky FM, Hattersley AT. Insights into the biochemical and genetic basis of glucokinase activation from naturally occurring hypoglycemia mutations. Diabetes. 2003;52:2433-24340

11. Cuesta-Munoz AL, Huopio $H$, Otonkoski T, Gomez-Zumaquero JM, Nanto-Salonen K, Rahier J, Lopez-Enriquez S, Garcia-Gimeno MA, Sanz $P$, Soriguer FC, Laakso M. Severe persistent hyperinsulinemic hypoglycemia due to a de novo glucokinase mutation. Diabetes. 2004; 53:2164-2168

12. Wabitsch $M$, Lahr $G$, Van de Bunt $M$, Marchant $C$, Lindner $M$, von Puttkamer J, Fenneberg A, Debatin KM, Klein R, Ellard S, Clark A, Gloyn AL. Heterogeneity in disease severity in a family with a novel G68V GCK activating mutation causing persistent hyperinsulinaemic hypoglycaemia of infancy. Diabet Med. 2007;24:1393-1399.

13. Duvanel CB, Fawer CL, Cotting J, Hohlfeld P, Matthieu JM: Long-term effects of neonatal hypoglycemia on brain growth and psychomotor development in small-for-gestational-age preterm infants. $J$ Pediatr. 1999;134:492-498

14. Matschinsky FM: Regulation of pancreatic $\beta$-cellglucokinase: from basics to therapeutics. Diabetes. 2002;51(3):394-404

15. Davis EA, Cuesta-Munoz A, Raoul M, Buettger C, Sweet I, Moates M, Magnuson MA, Matschinsky FM. Mutants of glucokinase cause hypoglycaemia- and hyperglycaemia syndromes and their analysis illuminates fundamental quantitative concepts of glucose homeostasis. Diabetologia. 1999;42:1175-1186

16. Froguel $P$, Zouali $H$, Vionnet $N$, Velho $G$, Vdaire $M$, Sun $F$, Lesage $S$, Stoffel M, Takeda J, Passa P, Permutt A, Beckmann JS, Bell GI, Cohen D: Familial hyperglycemia due to mutations in glucokinase. $N$ Engl J Med. 1993;328:697 -702 
17. Njolstad PR, Sovic O, Cuesta-Munoz A, Bjorkhaug L, Massa O, Barbetti F, Undlien DE, Shiota C, Magnuson MA, Molven A, Matschinsky FM, Bell GI: Neonatal diabetes mellitus due to complete glucokinase deficiency. $N$ Engl J Med. 2001;344:1588-1592

18. Dullaart RP, Hoogenberg K, Rouwe CW, Stulp BK. Family with autosomal dominant hyperinsulinism associated with A456V mutation in the glucokinase gene. J Intern Med. 2004;255:143-145

19. Matschinsky FM, Magnuson MA, Zelent D, Jetton TL, Doliba N, Han Y, Taub R, Grimsby J. The network of glucokinase-expressing cells in glucose homeostasis and the potential of glucokinase activators for diabetes therapy. Diabetes. 2006;55:1-12 